\title{
Criticality in Vibrated Frictional Flows at a Finite Strain Rate
}

\author{
Geert Wortel, ${ }^{1}$ Olivier Dauchot, ${ }^{2}$ and Martin van Hecke ${ }^{1,3}$ \\ ${ }^{1}$ Huygens-Kamerlingh Onnes Lab, Leiden University, P.O. Box 9504, 2300 RA Leiden, The Netherlands \\ ${ }^{2}$ EC2M, UMR Gulliver 7083 CNRS, ESPCI ParisTech, PSL Research University, 10 rue Vauquelin, 75005 Paris, France \\ ${ }^{3}$ FOM Institute AMOLF, Science Park 104, 1098 XG Amsterdam, The Netherlands
}

(Received 18 March 2016; revised manuscript received 12 July 2016; published 3 November 2016)

\begin{abstract}
We evidence critical fluctuations in the strain rate of granular flows that are weakly vibrated. Strikingly, the critical point arises at finite values of the mean strain rate and vibration strength, far from the yielding critical point at a zero flow rate. We show that the global rheology, as well as the amplitude and correlation time of the fluctuations, are consistent with a mean-field, Landau-like description, where the strain rate and the stress act as conjugated variables. We introduce a general model which captures the observed phenomenology and argue that this type of critical behavior generically arises when self-fluidization competes with friction.
\end{abstract}

DOI: 10.1103/PhysRevLett.117.198002

Fluctuations play an essential role in flows of disordered media [1-17]. In the simplest scenario, such fluctuations are rate independent, as in thermal systems or strongly vibrated granular flows [16-18]. New phenomena arise when fluctuations are generated by the flow itself, as observed for emulsions [5], foams [6], and granular matter [7-13]. Self-fluidization is particularly spectacular for granular media: for example, the finite yield threshold, a hallmark of static granular media, completely vanishes in the presence of flow anywhere in the granulate [9-13]. The reasons are, first, that the particles are so hard that tiny motions cause large fluctuations in the contact forces $[8,19]$ and, second, that sliding friction is nearly rate independent. Self-fluidization also qualitatively modifies the flowing properties of granular media; when the imposed stress is lowered, the flow is found to stop discontinuously: stresscontrolled granular flows have a nonzero minimal flow rate $[10,20,21]$.

What is the precise role of fluctuations for granular flows? Can local fluctuations organize in strong and slow collective fluctuations? How can we model the mutual coupling between fluctuations and flow? To answer these questions, we probe the fluctuations and the flow of weakly vibrated granular media sheared in a vertically vibrated split-bottom cell $[9,10,22]$. Here, the flow is driven by the rotation of a disk at the bottom of the cell, with the rate quantified by the dimensionless parameter $S$ - to be precisely defined below-while the dimensionless driving torque $T$ is measured [Fig. 1(a)]. For the low vibration strength $\Gamma$, the flow curves $T(S)$ exhibit three distinct regimes [Fig. 1(a)]: a vibration dominated flow regime (low $S$, positive slope) and an inertial flow one (large $S$, positive slope) separated by a self-fluidized flow one (intermediate $S$, negative slope). However, for vibration strengths $\Gamma$ larger than a critical vibration strength $\Gamma_{c}$, the self-fluidized flow regime disappears [10].
In this Letter, we report on a new and crucial experimental fact illustrated in Figs. 1(b) and 1(c). First, by controlling the driving torque $T$ and measuring the timeresolved rotation rate $S(t)$, we reveal that, decreasing $\Gamma$ towards $\Gamma_{c}$, fluctuations in $S$ become increasingly large and slow when $(T, \Gamma) \rightarrow\left(T_{c}, \Gamma_{c}\right)$ [Figs. 1(b) and 1(c)]. Second, we show that the flow curves $T(S)$, obtained at a fixed $S$, and their variation as a function of $\Gamma$ can be captured in a mean-field-type expansion around $\left(T_{c}, \Gamma_{c}\right)$. Together, these experiments evidence the existence of a nonzero or finite flow-rate (FFR) critical point. While strong fluctuations have been studied near the zero flow-rate yielding point [2], we stress that critical fluctuations at FFR critical points have not been reported before, presumably because they remain hidden in the absence of an external source of vibrations. Finally, we introduce a general model that combines a microscopic frictional rheology with fluctuations of the microscopic stresses. This model successfully describes the experimentally observed $\Gamma$-dependent rheology and the emergence of the FFR critical point, naturally capturing the intricate coupling between stress, flow rate, and fluctuations. Our results suggest that the FFR critical point is robust and that similar critical behavior may arise in other frictional or nearly rate-independent systems, leading to potentially hazardous fluctuations in previously overlooked flow regimes.

Setup and phenomenology.-The widely studied splitbottom geometry $[9,10,14,22]$ produces smooth and well controlled granular flows. Our cell consists of an acrylic cylindrical container (inner radius $7.0 \mathrm{~cm}$ ), at the bottom of which a rotating disk of radius $r_{s}$ of $4 \mathrm{~cm}$ drives the flow. The roughness of the top surface of the disk and the cylinder bottom ensures no-slip boundary conditions. The cell is filled to a height $h$ of $24 \mathrm{~mm}$ with black soda-lime glass beads with diameters $d=1.15 \pm 0.15 \mathrm{~mm}$. For such a filling height, the flow is a vertical shear band located 

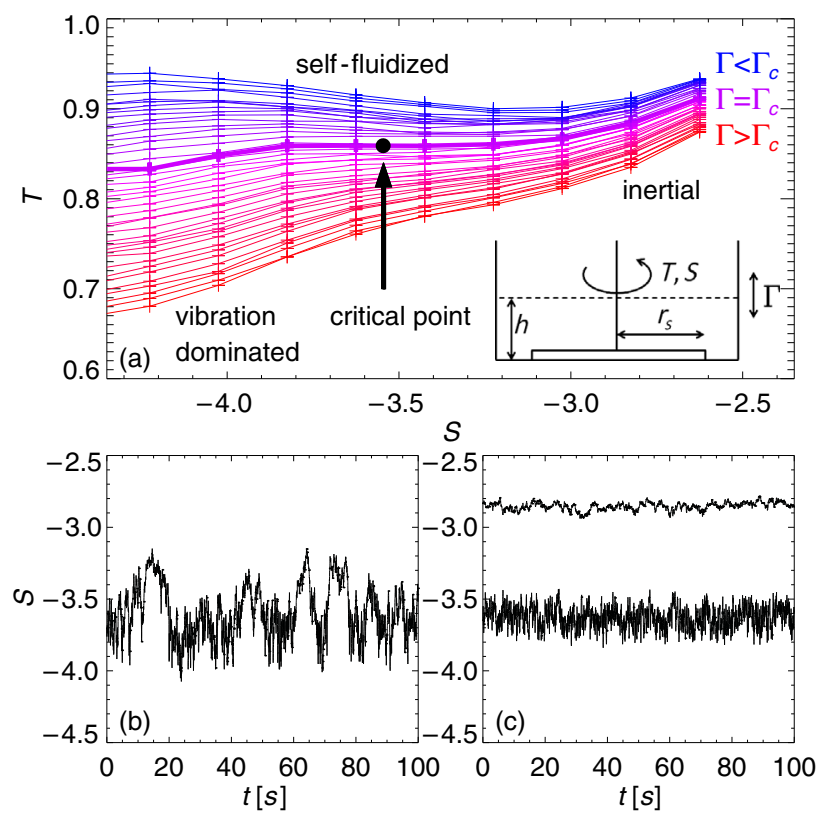

FIG. 1. (a) Flow curves obtained in a vibrated granular media sheared in a split-bottom cell: dimensionless torque $T$ as function of the rotation rate expressed by the dimensionless parameter $S$ (see the setup and phenomenology section for a precise definition), for a range of vibration strength $\Gamma \in[0.3,0.7]$. For $\Gamma<\Gamma_{c} \approx 0.46$, the flow curves exhibit a minimum. (Inset) Schematic of a vibrated split-bottom shear cell, indicating the important parameters. (b),(c) Rotation rate fluctuations $S(t)$ detected in torque-controlled experiments. (b) Strong and slow fluctuations close to the finite flow-rate (FFR) critical point $\left(S_{c}, T_{c}\right)$ indicated in (a) $[(T, \Gamma)=(0.84,0.71)]$. (c) Small fluctuations away from the critical point [top, $(T, \Gamma)=(0.85,0.71)$; bottom, $(T, \Gamma)=(0.79,0.92)]$. Note that, in torque-controlled experiments, $\Gamma_{c} \approx 0.65$ (see the text).

above the split in the bottom. An Anton Paar DSR 301 rheometer, which can be employed in rate- or torquecontrolled mode, sets the dimensional driving torque $\tilde{T}$ or the rotation rate $\Omega$. We vertically vibrate the system as $A \sin (2 \pi f t)$, with $f=63 \mathrm{~Hz}$, and control the dimensionless vibration strength $\Gamma=A(2 \pi f)^{2} / g$, where $g$ is the gravitational acceleration. The disk rotation is probed with an optical angular encoder (Heidenhain ERO 2500) directly coupled to the disk. We express our results in dimensionless units $T:=\tilde{T} / \tilde{T}_{y}$, where $\tilde{T}_{y}$ is the dynamic yield torque ( $T$ in the limit $S \rightarrow-\infty$ ) in the absence of external vibrations and $S:=\log (I)$, where $I=\Omega d /(g h)^{1 / 2}$ is the so-called apparatus scale inertial number [3].

We note that, in torque-controlled experiments, $\Gamma_{c}=0.65 \pm 0.01$-as will be determined from both the zero slope inflection point of the torque-controlled flow curves and the divergence of fluctuations-while, in ratecontrolled experiments, $\Gamma_{c}=0.46 \pm 0.01$, as will be determined from the zero slope inflection point of the rate-controlled flow curves. We believe this difference to be due to the complex combination of large intrinsic
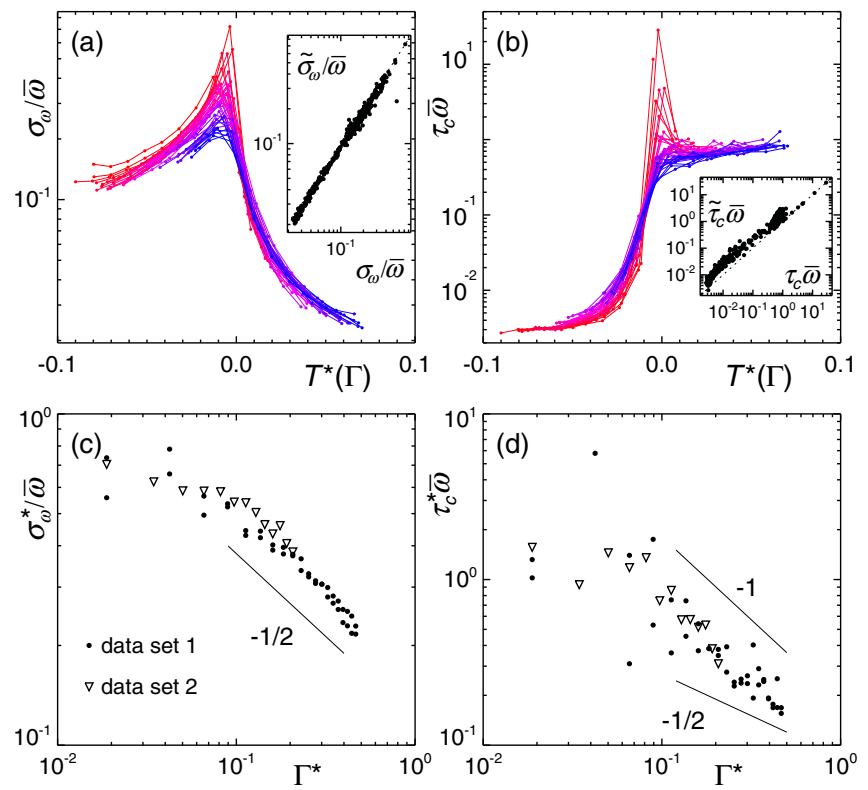

FIG. 2. Critical fluctuations. (a),(b) Fluctuation magnitude $\sigma_{\omega} / \bar{\omega}$ as a function of $T^{*}$ for $\Gamma \in[0.65,0.94]$. (Inset) $\tilde{\sigma}_{\omega}$ and $\sigma_{\omega}$ are essentially equal. (b) Correlation time $\tau_{c} \overline{\bar{\omega}}$ as a function of $T^{*}$. (Inset) $\tilde{\tau}_{c}$ scales linearly with $\tau_{c}$. (c),(d) Evidence for critical scaling of, respectively, $\sigma_{\omega}^{*}$ and $\tau_{c}^{*}$ with $\Gamma^{*}$ in two data sets taken several weeks apart.

fluctuations, finite size effects, and the nonperfect feedback loop of the rheometer in rate-controlled experiments.

Critical fluctuations. - We first perform experiments at constant torque $T$ and vibration strength $\Gamma>\Gamma_{c}$ and determine the magnitude and correlation time of the fluctuations in rotation rate via the instantaneous angular position $\theta(t)$ of the bottom disk. We extract the rotation rate $\omega(t):=\partial_{t} \theta(t)$, after carefully checking to see that $\theta(t)$ is probed at sufficiently high temporal resolution. We then compute the averaged rotation rate $\bar{\omega}=\langle\omega\rangle$, the amplitude of its fluctuations $\sigma_{\omega}^{2}=\left\langle\delta \omega^{2}\right\rangle$, and the temporal correlations $R(\tau)=\langle\delta \omega(t+\tau) \delta \omega(t)\rangle / \sigma_{\omega}^{2}$, where $\delta \omega=\omega-\bar{\omega}$ and $\langle\cdot\rangle$ denote temporal averages. The correlation time $\tau_{c}$ is extracted by fitting the autocorrelation to an exponential (and is consistent with the time obtained by integrating the correlation function). Figures 2(a) and 2(b) display the resulting dimensionless fluctuation amplitude $\sigma_{\omega} / \bar{\omega}$ and the dimensionless correlation time $\tau_{c} \bar{\omega}$ as a function of the relative torque $T^{*}(\Gamma)=\left(T-T_{i}(\Gamma)\right) / T_{i}(\Gamma)$, where $T_{i}(\Gamma)$ is the inflection point observed in the flow curves, which separates the vibration dominated flow regime, from the fast inertial flow regime, for values of $\Gamma>\Gamma_{c}$. There is a sharp contrast between the fluctuations at either side of the peaks: the vibration dominated flow regime has strong but short-time correlated fluctuations, while the fast inertial flow regime has small fluctuations with a time scale $\approx \bar{\omega}^{-1}$. Crucially, there is a sharp transition between these regimes: both the fluctuation amplitude $\sigma_{\omega} / \bar{\omega}$ and the correlation time $\tau_{c} \bar{\omega}$ exhibit a sharp 
maximum at $T^{*} \simeq 0$, which rapidly grows when $\Gamma^{*}=\left(\Gamma-\Gamma_{c}\right) / \Gamma_{c} \rightarrow 0^{+}$. The peak in the rotation rate fluctuations diverges in a manner consistent with a power-law scaling $\sim \Gamma^{*-\tilde{\gamma}}$, with $\tilde{\gamma} \approx 0.5$ [Fig. 2(c)]. The correlation times are too noisy to be reliably fitted to a power law $\Gamma^{*-\mu}$, but, if any, $\mu \in[0.5,1]$ [Fig. 2(d)]. Together, these signals provide strong evidence for critical behavior. To check the robustness of our measurements, we also determine the fluctuation magnitude and the correlation by considering the rotating disk as a massive random walker with drift. We thus characterize the mean square angular displacement $\Delta \theta(t)^{2}=\left\langle\left(\theta\left(t_{0}+t\right)-\theta\left(t_{0}\right)\right)^{2}\right\rangle$. The amplitude of the rotation rate fluctuations $\tilde{\sigma}_{\omega}$ and the correlation time $\tilde{\tau}_{c}$ are then extracted from the asymptotics: for $t / \tilde{\tau}_{c} \ll 1$, the dynamics is ballistic with $\Delta \theta(t)^{2} \sim \tilde{\sigma}_{\omega}^{2} \tau$, while, for $t / \tilde{\tau}_{c} \gg 1$, it is diffusive, with $\Delta \theta(t)^{2} \sim 2 \tilde{\sigma}_{\omega}^{2} \tau_{c} t$. These two independent protocols yield consistent results, as shown in the insets of Figs. 1(a) and 1(b).

Scaling of the flow curves. - The critical behavior reported above suggests that the torque and the rotation rate should be related via a Landau expansion in the critical regime:

$$
T=a\left(S-S_{i}\right)^{3}+b\left(S-S_{i}\right)+T_{i},
$$

where $\left(T_{i}(\Gamma), S_{i}(\Gamma)\right)$ are the inflection points of the flow curves. In order to probe this relation, we perform ratecontrolled experiments in which we can also access the negative slope regime. The flow curves, shown in Fig. 1(a), are indeed reminiscent of a third order polynomial. Fitting the data accordingly, we extract $\left(S_{i}, T_{i}\right), a$ and $b$, and the local maximum $\left(S_{+}, T_{+}\right)$and minimum $\left(S_{-}, T_{-}\right)$as a function of $\Gamma$. As shown in Fig. 3(a), the flow curves can be rescaled on two distinct branches, below and above $\Gamma_{c}$, over a substantial range. As expected, the cubic coefficient $a$ remains essentially constant ( $a \simeq 2$; not shown here). The coefficient $b$, which sets the slope at the inflection point, akin to an inverse susceptibility $\chi^{-1}$, crosses zero at $\Gamma=\Gamma_{c}$ and increases linearly with $\Gamma^{*}$. The location of the extrema $\left(S_{ \pm}, T_{ \pm}\right)$in the $(S, \Gamma)$ and $(T, \Gamma)$ planes, displayed in Figs. 3(c) and 3(d), together with the location of the inflection point $\left(S_{i}, T_{i}\right)$, determines the so-called spinodal lines, which are the stability limits of the fast and slow flow phases. The region of "coexistence" corresponds here to the set of parameters for which the flow curves have a negative slope. The width of this region $\Delta=S_{+}-S_{-}$scales like $\left|\Gamma^{*}\right|^{\beta}$, with $\beta=0.5$ for $\Gamma^{*}<0$, in agreement with Eq. (1) and the linear dependence of $b$ with $\Gamma^{*}$.

FFR critical point.-Our data for both torque-controlled and rate-controlled experiments provide strong evidence for the existence of a critical point at a finite flow rate, characterized by the following scaling relations:

$$
\Delta \sim \Gamma^{* \beta}, \quad \beta \simeq 0.5
$$
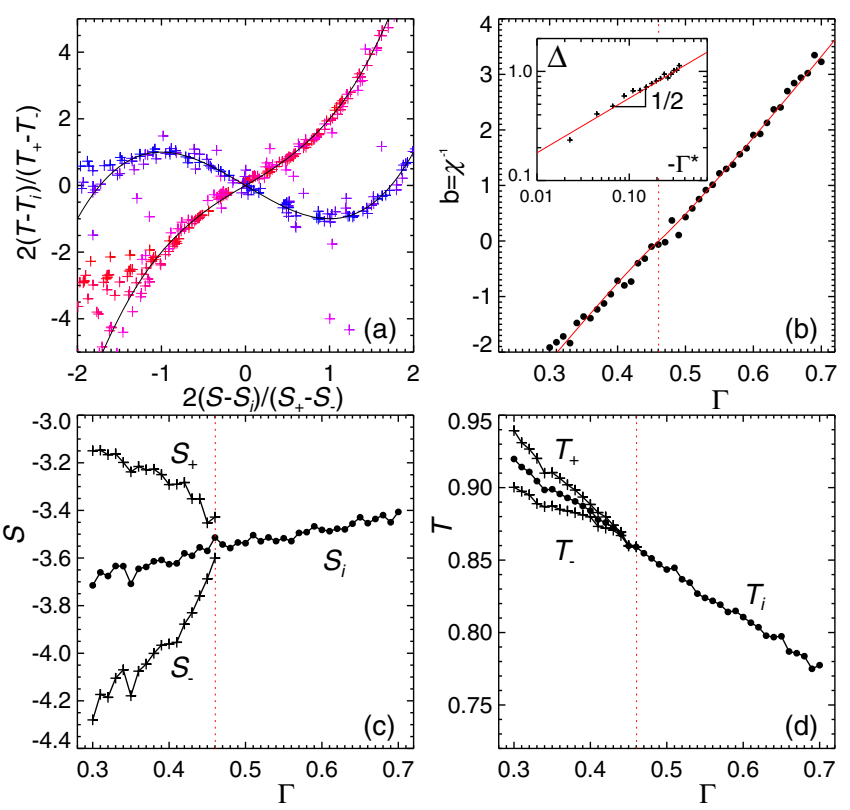

FIG. 3. (a) Flow curve data collapsed onto master curves for $\Gamma \in[0.3,0.7]$. (b) Inverse susceptibility $\chi^{-1}$ vs $\Gamma$. (Inset) Log-log plot of $\Delta=S_{+}-S_{-}$as a function of $-\Gamma^{*}$. (c),(d) Location of the extrema $\left(S_{ \pm}, T_{ \pm}\right)$and the inflection point $\left(S_{i}, T_{i}\right)$ in the planes $(\Gamma, S)$ and $(\Gamma, T)$. The vertical dashed line indicates $\Gamma_{c}$.

$$
\begin{array}{cc}
\chi \sim \Gamma^{*-\gamma}, & \gamma \simeq 1 \\
\sigma_{\omega} / \bar{\omega} \sim \Gamma^{*-\tilde{\gamma}}, & \tilde{\gamma} \simeq 0.5 \\
\tau_{c} \bar{\omega} \sim \Gamma^{*-\mu}, & \mu \in[0.5,1] .
\end{array}
$$

The fact that the critical behavior of $\chi$, obtained for averaged quantities in rate-controlled experiments, and that of $\sigma_{\omega}^{2}$, obtained from the fluctuations in torque-controlled experiments, coincide is a strong indication of the relevance of our analysis. Both the value of the exponents and the quality of the description of the flow curves by Eq. (1) suggest that a mean-field description should capture the essence of the observed phenomenology.

Flow model.-Finally, we introduce a general fluctuationfrictional (FF) model that captures the observed rheology. We combine a frictional local rheology with fluctuations that are induced by both vibrations and flow, and we show that the average rheology of this model exhibits all of the experimentally observed hallmarks, including the FFR critical point. First, we introduce an agitation strength A, which is a function of both flow-induced and vibrationinduced agitations [23]:

$$
A=A_{g}(\Gamma, I),
$$

where $A_{g}=0$ only when both $\Gamma$ and $I$ are zero. Second, we postulate that the local stresses $T_{m}$ are fluctuating around 

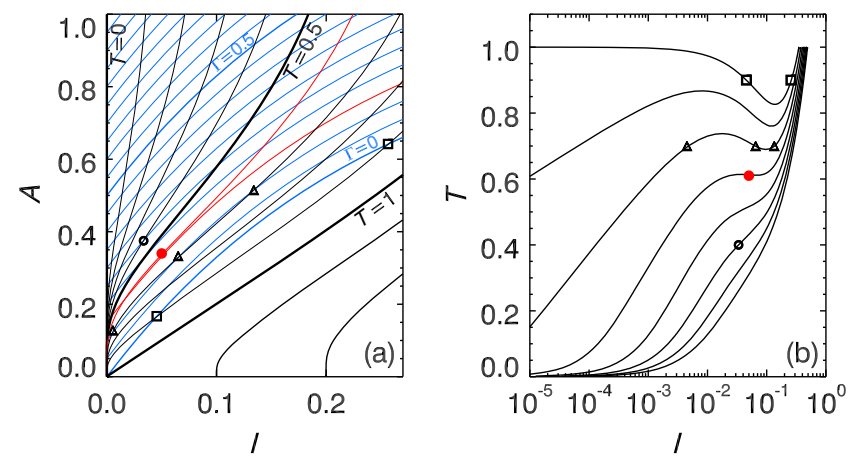

FIG. 4. FF model (for details, see Ref. [24]). (a) Contour curves of constant $\Gamma$ (blue) and constant $T$ (black). Their intersections, for example at $(\Gamma, T)=(0.25,0.4)$ (the open circle), $(0.1,0.7)$ (the three triangles), and $(0,0.9)$ (the two squares) determine $T(I)$. The red contour curves for $T=0.6$ and $\Gamma=0.15$ are (nearly) tangent, leading to the critical point (the red filled circle). (b) Corresponding flow curves for $\Gamma=0,0.05, \ldots, 0.35$ (ordered from top to bottom) - the circles, squares and boxes match those in (a).

their mean $T$, and that the microscopic stress distribution $P\left(T_{m}\right)=\bar{P}\left(\left(T_{m}-T\right) / A\right)$, where $\bar{P}(x)$ is a given normalized distribution centered at $x=0$-note that $A$ sets the width of $P\left(T_{m}\right)$. Third, we determine the global flow rate $I$ as the mean of the microscopic flow rates $I_{m}$, where $I_{m}$ and $T_{m}$ are related by simple frictional Herschel-Bulkley rheology with a finite yield stress-in particular, $I_{m}=0$ when $\left|T_{m}\right|<1$. Combining these ingredients, we find

$$
I(A, T)=\int_{-\infty}^{\infty} \frac{d T_{m}}{A} \bar{P}\left(\frac{T_{m}-T}{A}\right) I_{m}\left(T_{m}\right) .
$$

For a prescribed set of functions $A_{g}, P$, and $I_{m}\left(T_{m}\right)$, Eqs. (6) and (7) completely set the flow curves $T(I, \Gamma)$. We start with a definite choice of $A_{g}, \bar{P}$, and $I_{m}$ [24], then show that our conclusions are insensitive to this choice (details are provided in the Supplemental Material [25]).

We now show that the FF model captures all of the qualitative features of the rheology of the weakly vibrated flows. First, it exhibits the experimentally observed singularity of the stress $T(I, A)$ at the origin: when $A=0$, Eq. (7) implies that $T_{m}=T, I_{m}=I$, so that the macrorheology is identical to the microscopic rheology and $T(0+, 0)=1$. In contrast, when $A>0$ but $I=0$, Eq. (7) implies that the stress distribution must be symmetric around zerotherefore, $T=0$ and, in particular, $T\left(0,0^{+}\right)=0$. The model thus captures the discontinuous vanishing of the yield stress when $\Gamma$ becomes finite. To proceed further, the solutions to this model can be understood by considering the variation of $\Gamma$ and $T$ in the $(I, A)$ plane (Fig. 4). The flow curves $T(I, \Gamma)$ can be determined graphically via the intersections of the contour curves of $T$ and $\Gamma$ by fixing $\Gamma$, varying $T$, and determining the corresponding value(s) of $I$. For $T>1$ there is only one intersection, corresponding to rapid flows, and in the remainder we focus on $T \leq 1$. (i) For a large $\Gamma$, there is only one intersection (the black dot), leading to monotonic flow curves $T(I)$. (ii) For a small $\Gamma$, there are three intersections (the crosses), corresponding to nonmonotonic flow curves. (iii) In between these two regimes is the critical $\Gamma_{c}$ curve (red), for which the three intersection points merge (the red dot). (iv) Finally, for $\Gamma=0$, there are precisely two intersections, corresponding to the only flow curve that has a finite $T$ and a negative slope at $I=0$. We stress that the scenario that emerges captures the essence of the experimental flow curves shown in Fig. 1(a), without having to make any assumptions about the behavior of $A$ near the FFR critical point.

Clearly, the essence of this scenario does not depend on the details of the agitation function $A_{g}$, the distribution $\bar{P}$, or the local rheology $T_{m}$. The only condition is that the $\Gamma=0$ curve is steeper than the $T=1$ contour at the origin, such that there are two intersections between the $\Gamma=0$ and $T=1$ curves (for other examples of flow curves, including occasions when this condition is violated, see the Supplemental Material [25]).

Discussion.-We have uncovered a dynamical critical point in agitated frictional flows. We stress again that the concomitant large fluctuations arise at finite flow rates, away from the yielding point where strong fluctuations were seen before [2], which makes this an experimentally easily accessible yet nontrivial critical point which deserves further investigation. We have argued that this criticality emerges from the interplay of external vibration and selffluidization. Both act as sources of agitation, but they influence the rheology very differently: while external agitations set the yield stress to zero and impose a positive slope on the limit of zero flow rate, flow-induced fluctuations cause a negative slope in the flow curves, at least in the absence of externally provided fluctuations. The FFR critical behavior emerges due to the competition of these two sources of agitation.

From a more theoretical point of view, the model we have introduced here is purely phenomenological and is essentially mean field. An alternative, more complete strategy would be to write down a dynamical equation for the microscopic stress distribution, as introduced in the Hébraud-Lequeux [1] and related fluidity models [26,27], which at present capture neither the critical scaling of the flow curves nor the diverging fluctuations. In these models the self-fluidization is captured by a diffusion term for the local stresses, the amplitude of which is linearly related to the amount of stress exceeding the local yield stress. Adding any finite amount of external noise via a constant term in the diffusion amplitude, we expect the dynamical yield stress to vanish as observed here. Then, following Ref. [27], one could work out the relation between the model parameters and the flow rate and, hopefully, obtain the observed nonmonotonic rheology. Stress fluctuations 
could also be taken into account following the very recent work of Agoritsas et al. [28]. It is an open question whether such models could exhibit a negative slope at zero flow rate in the absence of external vibrations.

We thank J. Dijksman, J. Mesman, H. Eerkens, E. Agoritsas, E. Bertin, and K. Martens for the discussions, technical support, and early experiments, and Stichting voor Fundamenteel Onderzoek der Materie/Nederlandse Organisatie voor Wetenschappelijk Onderzoek for the funding.

[1] P. Hébraud and F. Lequeux, Phys. Rev. Lett. 81, 2934 (1998).

[2] D. Howell, R. P. Behringer, and C. Veje, Phys. Rev. Lett. 82, 5241 (1999).

[3] GDR MiDi Collaboration, Eur. Phys. J. E 14, 341 (2004).

[4] O. Pouliquen and R. Gutfraind, Phys. Rev. E 53, 552 (1996).

[5] J. Goyon, A. Colin, G. Ovarlez, A. Ajdari, and L. Bocquet, Nature (London) 454, 84 (2008).

[6] G. Katgert, B. P. Tighe, M. E. Möbius, and M. van Hecke, Europhys. Lett. 90, 54002 (2010).

[7] K. A. Reddy, Y. Forterre, and O. Pouliquen, Phys. Rev. Lett. 106, 108301 (2011).

[8] M. van Hecke, C.R. Phys. 16, 37 (2015).

[9] K. Nichol, A. Zanin, R. Bastien, E. Wandersman, and M. van Hecke, Phys. Rev. Lett. 104, 078302 (2010); K. Nichol and M. van Hecke, Phys. Rev. E 85, 061309 (2012).

[10] J. A. Dijksman, G. H. Wortel, L.T. H. van Dellen, O. Dauchot, and M. van Hecke, Phys. Rev. Lett. 107, 108303 (2011); G. H. Wortel, J. A. Dijksman, and M. van Hecke, Phys. Rev. E 89, 012202 (2014).

[11] K. Kamrin and G. Koval, Phys. Rev. Lett. 108, 178301 (2012); D. L. Henann and K. Kamrin, Proc. Natl. Acad. Sci. U.S.A. 110, 6730 (2013); Phys. Rev. Lett. 113, 178001 (2014).

[12] M. Bouzid, M. Trulsson, P. Claudin, E. Clement, and B. Andreotti, Phys. Rev. Lett. 111, 238301 (2013).
[13] Y. Forterre and O. Pouliquen, Annu. Rev. Fluid Mech. 40, 1 (2008).

[14] G. H. Wortel and M. van Hecke, Phys. Rev. E 92, 040201(R) (2015).

[15] P. Schall and M. van Hecke, Annu. Rev. Fluid Mech. 42, 67 (2010).

[16] G. Caballero-Robledo and E. Clément, Eur. Phys. J. E 30, 395 (2009).

[17] J. Javier Brey, M. J. Ruiz-Montero, and F. Moreno, Phys. Rev. E 63, 061305 (2001).

[18] X. Jia, T. Brunet, and J. Laurent, Phys. Rev. E 84, 020301 (2011).

[19] P. Umbanhowar and M. van Hecke, Phys. Rev. E 72, 030301 (2005).

[20] H. Jaeger and Chu-Heng Liu, S. Nagel, and T. Witten, Europhys. Lett. 11, 619 (1990).

[21] O. Kuwano, T. Hatano, and R. Ando, Geophys. Res. Lett. 40, 1295 (2013).

[22] D. Fenistein, J. W. van der Meent, and M. van Hecke, Phys. Rev. Lett. 92, 094301 (2004); 96, 118001 (2006); , 6, 2901 (2010).

[23] We use $I$ rather than $S$ in the model, as we want to consider the absence of flow at $I=0$.

[24] We take $A_{g}(I, \Gamma)=\Gamma+[1-\exp (4 I)]$, which captures that both vibrations and flow induce fluctuations which saturate for large flow rates, $\bar{P}(x)=1 / 2 \exp (-|x|)$, and $T_{m}$ follows the Herschel-Bulkley rheology: $T_{m}=\operatorname{sign}\left(I_{m}\right)\left[1+\left|I_{m}\right|\right]$, with $I_{m}=0$ when $\left|T_{m}\right|<1$.

[25] See Supplemental Material at http://link.aps.org/ supplemental/10.1103/PhysRevLett.117.198002 for a detailed discussion of the FF model and other examples of flow curves.

[26] L. Bocquet, A. Colin, and A Ajdari, Phys. Rev. Lett. 103, 036001 (2009).

[27] V. Mansard, A. Colin, P. Chauduri, and L. Bocquet, Soft Matter 7, 5524 (2011).

[28] E. Agoritsas, E. M. Bertin, K. Martens, and J.-L. Barrat, Eur. Phys. J. E 38, 71 (2015). 